\section{Ultrawideband monocycle pulse generation using dual-output intensity modulator}

\author{
Yue Peng, Hongming Zhang, Qingwei Wu, Xin Fu, and \\ Minyu Yao \\ State Key Laboratory on Integrated Optoelectronics, \\ Tsinghua University, Tsinghua National Laboratory for \\ Information Science and Technology, Department of \\ Electronic Engineering, Beijing 100084, China \\ E-mail: y-peng05@mails.tsinghua.edu.cn
}

Abstract. An approach to generate ultrawideband (UWB) monocycle pulses is proposed and experimentally demonstrated, based on a dual-output intensity modulator and tunable optical time delay. Positive and negative pulses are obtained from two output ports of the modulator, respectively, and are coupled together through different time delays. The generated monocycle pulse has a $10-\mathrm{dB}$ bandwidth of $6.5 \mathrm{GHz}$ and a central frequency of $3.7 \mathrm{GHz}$. ๑ 2008 Society of Photo-Optical Instrumentation Engineers.

[DOI: 10.1117/1.2938834]

Subject terms: ultrawideband; dual-output intensity modulator; monocycle pulse; polarization interferometer; microwave photonics.

Paper 080080LRR received Jan. 31, 2008; revised manuscript received Apr. 26, 2008; accepted for publication Apr. 28, 2008; published online Jun. 10, 2008.

\section{Introduction}

Ultrawideband (UWB) is a promising technology for shortrange wireless indoor communication due to many advantages, such as extremely wide bandwidth, low-power spectral density, and multipath immunity. ${ }^{1}$ The United States Federal Communications Commission (FCC) regulates the 7.5-GHz frequency band from 3.1 to $10.6 \mathrm{GHz}$ for unlicensed use of UWB with a power spectral density emission limit of $-41.3 \mathrm{dBm} / \mathrm{MHz}^{2}$ As a development of FTTx, UWB-over-fiber combines UWB and fiber access networks for broadband indoor wireless access. In recent years, the generation of UWB signals directly in the optical domain has attracted research interests, and many schemes have been proposed, such as UWB pulse generation based on cross-gain modulation $(\mathrm{XGM})^{3,4}$ or gain saturation effect ${ }^{5}$ in a semiconductor optical amplifier (SOA); phase modulation to intensity modulation (PM-IM) conversion using an optical frequency discriminator ${ }^{6-9}$; photonic microwave delay-line filter structures ${ }^{10}$; nonlinearly biased electrooptic intensity modulation ${ }^{11}$; spectral-shaping and dispersion-induced frequency-to-time conversion ${ }^{12}$; and optical polarization modulation with time delay. ${ }^{13,14}$

In this work, we propose and demonstrate a simple approach to optically generate a UWB monocycle pulse based on a dual-output electro-optic intensity modulator and optical delay line. The fundamental principle is that a dualoutput intensity modulator is applied by an electrical pulse, and then positive and negative pulses are obtained from

0091-3286/2008/\$25.00 @ 2008 SPIE two output ports, respectively, and coupled together through different time delays. Through adjusting the electrical pulse width and optical delay-line length, various UWB monocycle pulses can be generated. In our experimental demonstration, a UWB monocycle that has a $10-\mathrm{dB}$ spectrum bandwidth of $6.5 \mathrm{GHz}$ and central frequency of 43.7 $\mathrm{GHz}$ is acquired.

\section{Principle and Experiment}

The experiment setup of our proposed scheme is shown in Fig. 1(a). The dual-output electro-optic intensity modulator, which has been used for photonic analog-to-digital convertors $^{15}$ and microwave photonic filters, ${ }^{16}$ has two complementary output ports, i.e., the intensity of output 1 and output 2 is

$$
\begin{aligned}
& I_{1}(t)=\frac{1}{2} I_{i}\left\{1+\sin \left[\frac{\pi \cdot V_{s}(t)}{V_{\pi}}+\varphi_{b}\right]\right\}, \\
& I_{2}(t)=\frac{1}{2} I_{i}\left\{1+\sin \left[\frac{\pi \cdot V_{s}(t)}{V_{\pi}}+\varphi_{b}+\pi\right]\right\},
\end{aligned}
$$

where $I_{i}$ is the intensity of the distributed feedback (DFB) laser, $V_{s}$ is the applied electrical pulse, $V_{\pi}$ is the half-wave voltage of the modulator, and $\varphi_{b}$ is the initial bias phase. Choosing the proper $\varphi_{b}$ and amplitude of $V_{s}$, the modulator could work in the linear modulated region. Then

$$
I_{1}(t) \approx \frac{1}{2} I_{i}\left[1+\frac{\pi \cdot V_{s}(t)}{V_{\pi}}\right], \quad I_{2}(t) \approx \frac{1}{2} I_{i}\left[1-\frac{\pi \cdot V_{s}(t)}{V_{\pi}}\right],
$$

which shows that a positive optical pulse and negative optical pulse can be obtained from the two output ports, respectively, while electrical pulses are applied to the modulator. The complementary pulses are coupled after a tunable delay line (TDL). The combined signal is

$$
I_{o}(t)=I_{1}(t)+I_{2}(t-\tau) \approx I_{i}+\frac{1}{2} I_{i} \frac{\pi \cdot V_{s}(t)}{V_{\pi}}-\frac{1}{2} I_{i} \frac{\pi \cdot V_{s}(t-\tau)}{V_{\pi}},
$$

where $\tau$ is the time delay between two complementary pulses. Then by choosing a proper $\tau$, monocycle pulses can be detected by a photodetector (PD) at the coupler output.

In our experiment, a polarization interferometer based on a $\mathrm{LiNbO}_{3}$ phase modulator is used as a dual-output intensity modulator, whose principle is shown in Fig. 1(b). Adjusted by the polarization controller (PC) 1, input light is launched $45 \mathrm{deg}$ to the $x$ and $y$ axes of the phase modulator, by which the phase difference between the $x$ and $y$ directions will linearly change with the voltage of the electric signal. Two polarizations are made to interfere through a polarizing beamsplitter (PBS) as an in-line analyzer whose transmission axis is $\pm 45 \mathrm{deg}$ to the $x$ and $y$ axes. Then the intensity of output 1 and output 2 is complementary with orthogonal polarizations. Due to orthogonal polarizations of the two outputs, they will not interfere at the output of the coupler. The phase modulator is driven by a fixed pattern with a $622-\mathrm{MHz}$ repetition rate and 1:15 duty cycle, 


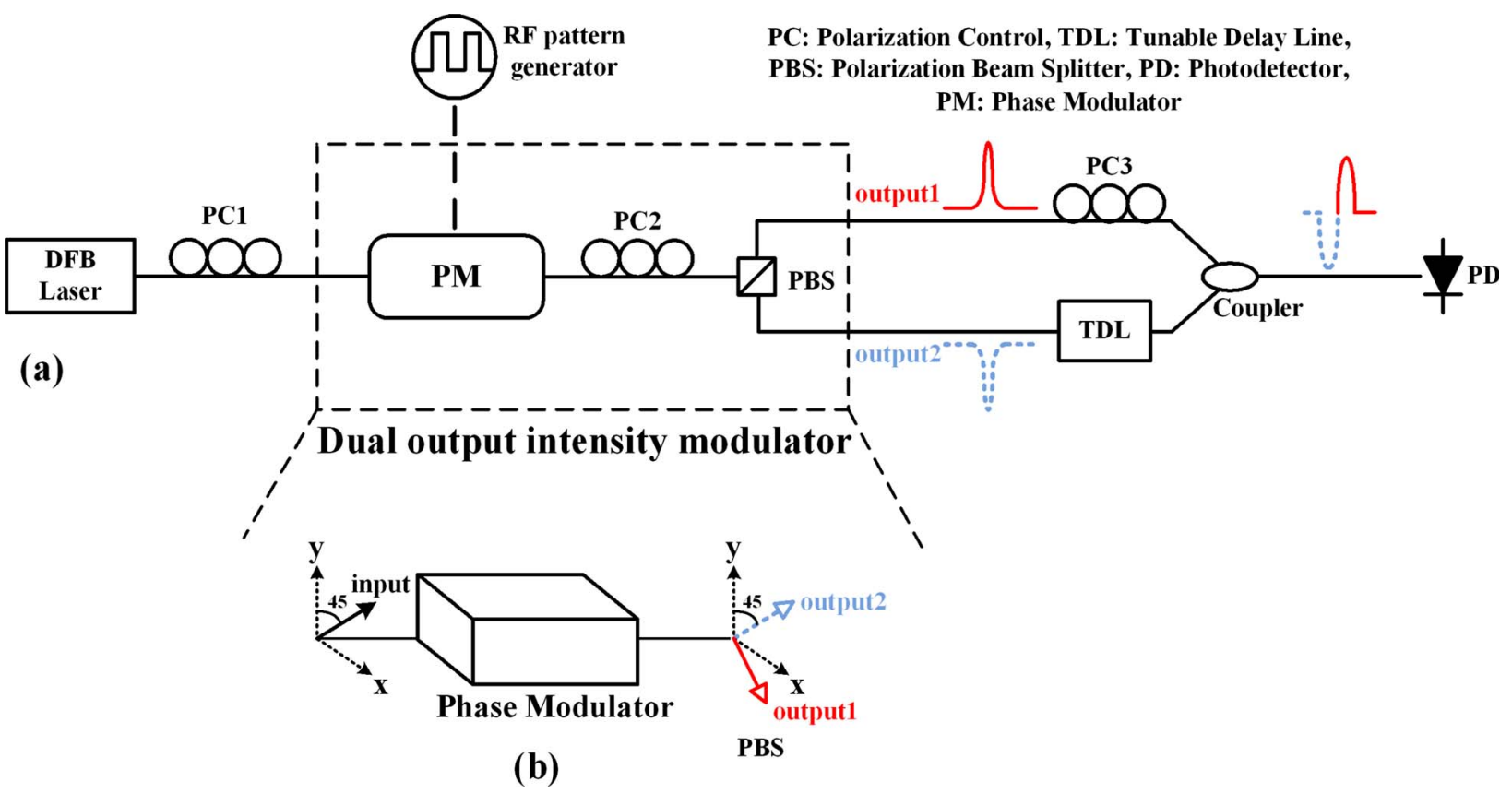

Fig. 1 (a) Experimental setup of proposed scheme. (b) Illustration of dual-output intensity modulator based on polarization interference.

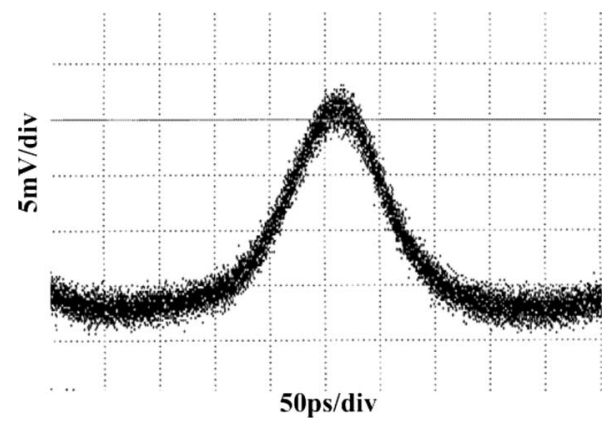

Fig. 2 Waveform of the optical positive pulse from output 1.

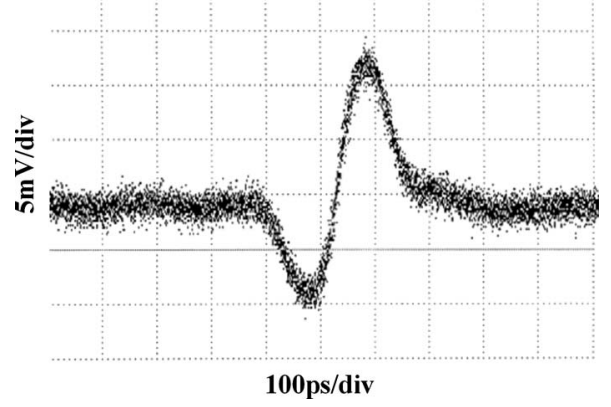

Fig. 3 Waveform of the generated monocycle pulse. and the optical pulse from output 1 is a Gaussian-like shape with a full width at half-maximum (FWHM) of $100 \mathrm{ps,} \mathrm{as}$ shown in Fig. 2.

A waveform of the generated monocycle pulse is shown in Fig. 3, which has a pulse width of about 130 ps. By controlling the TDL, a 0 or $\pi$ phase shift pulse can be generated. In Fig. 4, the RF spectrum, whose envelope represents the spectrum of the monocycle pulse, includes discrete frequency with an interval of $622 \mathrm{MHz}$, induced by the applied pattern signal. With the $10-\mathrm{dB}$ bandwidth at 6.5 GHz, and the central frequency at $3.7 \mathrm{GHz}$, then the calculated fractional bandwidth is $175 \%$.

By adjusting the electrical pulse width and optical delayline length, various UWB monocycle pulses can be generated. But the major disadvantage in our proposal is that the optical pulse width is limited by an applied electrical pulse, which restricts the frequency bandwidth of the UWB pulse.

\section{Conclusion}

A novel and simple approach to generate UWB monocycle pulses is proposed and experimentally demonstrated, based

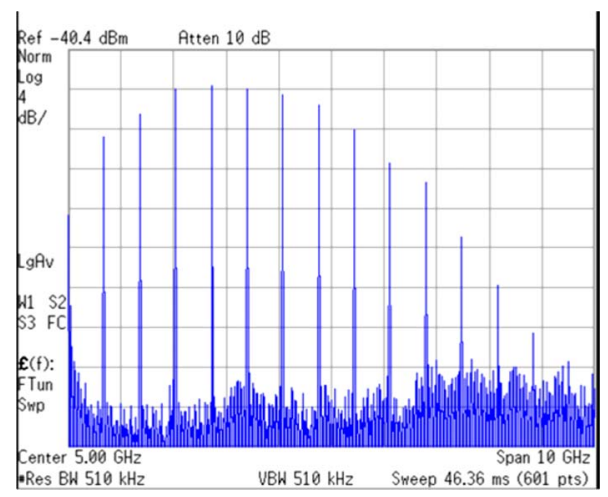

Fig. 4 RF power spectrum of the monocycle pulse. 
on a dual-output intensity modulator and optical tunable time delay. In our experiment, only one optical source is required, and a polarization interferometer based on a $\mathrm{LiNbO}_{3}$ phase modulator performs as a dual-output intensity modulator. By adjusting the optical tunable delay line, a monocycle pulse output can be realized by the combination of a positive optical pulse and a negative optical pulse. The result shows that the generated monocycle pulse has a $10-\mathrm{dB}$ bandwidth of $6.5 \mathrm{GHz}$ and central frequency of $3.7 \mathrm{GHz}$.

\section{Acknowledgments}

This work was supported by the National Natural Science Foundation of China (No. 60607008) and National 863 Program of China (No. 2007AA01Z271).

\section{References}

1. D. Porcine and W. Hirt, "Ultra-wideband radio technology: potential and challenges ahead," IEEE Commun. Mag. 41(7), 66-74 (2003).

2. G. R. Aiello and G. D. Rogerson, "Ultra-wideband wireless systems," IEEE Microw. Mag. 4(2), 36-47 (2003).

3. Q. Wang, F. Zeng, S. Blais, and J. Yao, "Optical ultrawideband monocycle pulse generation based on cross-gain modulation in a semiconductor optical amplifier," Opt. Lett. 31(21), 3083-3085 (2006).

4. J. Dong, X. Zhang, J. Xu, D. Huang, S. Fu, and P. Shum, "Ultrawideband monocycle generation using cross-phase modulation in a semiconductor optical amplifier," Opt. Lett. 32(10), 1223-1225 (2007).

5. J. Dong, X. Zhang, J. Xu, and D. Huang, "All-optical ultrawideband monocycle generation utilizing gain saturation of a dark return-to- zero signal in a semiconductor optical amplifier," Opt. Lett. 32(15), 2158-2160 (2007).

6. W. P. Lin and J. Y. Chen, "Implementation of a new ultrawide-band impulse system," IEEE Photonics Technol. Lett. 17(11), 2418-2420 (2005).

7. F. Zeng and J. Yao, "An approach to ultrawideband pulse generation and distribution over optical fiber," IEEE Photonics Technol. Lett. 18(7), 823-825 (2006).

8. F. Zeng and J. Yao, "Ultrawideband impulse radio signal generation using a high-speed electrooptic phase modulator and a fiber-Bragggrating-based frequency discriminator," IEEE Photonics Technol. Lett. 18(19), 2062-2064 (2006).

9. F. Zeng, Q. Wang, and J. Yao, "All-optical UWB impulse generation based on cross-phase modulation and frequency discrimination," Electron. Lett. 43(2), 121-122 (2007).

10. Q. Wang and J. Yao, "Switchable optical UWB monocycle and doublet generation using a reconfigurable photonic microwave delay-line filter," Opt. Express 15(22), 14667-14672 (2007).

11. Q. Wang and J. Yao, "UWB doublet generation using nonlinearlybiased electro-optic intensity modulator," Electron. Lett. 42(22), 1304-1305 (2006).

12. C. Wang, F. Zeng, and J. Yao, "All-fiber ultrawideband pulse generation based on spectral shaping and dispersion-induced frequency-totime conversion," IEEE Photonics Technol. Lett. 19(3), 137-139 (2007).

13. H. Chen, M. Chen, C. Qiu, J. Zhang, and S. Xie, "UWB monocycle pulse generation by optical polarisation time delay method," Electron. Lett. 43(9), 542-543 (2007).

14. H. Chen, M. Chen, J. Zhang, and S. Xie, "UWB monocycle and doublet pulses generation in optical domain," IEEE Intl. Top. Meet. Microwave Photon. MWP'07, pp. 145-148 (2007).

15. J. C. Twichell and R. Helkey, "Phase-encoded optical sampling for analog-to-digital converters," IEEE Photonics Technol. Lett. 12(9), 1237-1239 (2000).

16. D. B. Hunter, "Incoherent bipolar tap microwave photonic filter based on balanced bridge electro-optic modulator," Electron. Lett. 40(14), 856-858 (2004). 